\title{
Surveillance sanitaire estivale des populations piscicoles du Rhône au voisinage du Centre Nucléaire de Production d'Électricité (CNPE) du Tricastin. Synthèse 2008-2013
}

\section{Summer health monitoring of fish populations of the Rhône River near Tricastin nuclear power plant. Synthesis 2008-2013}

\author{
Patrick Girard ${ }^{1}$, Jean-Yves Brana ${ }^{2}$, Jean-François Fruget ${ }^{2^{\star}}$ \\ 1 Lotissement Super-Peynier, 13790 Peynier, France \\ 2 ARALEP, Campus LyonTech-La Doua, 66 Bld Niels Bohr, CS 52132, 69603 Villeurbanne cedex, France
}

Résumé - Une surveillance sanitaire des populations piscicoles du Rhône a été mise en place chaque été depuis 2005 en amont et en aval du CNPE du Tricastin dans le cadre de la surveillance préventive en cas d'aléa thermique impactant la production d'électricité. Elle s'appuie sur des descripteurs biologiques pertinents et validés (indice de condition, parasitisme) et sur deux outils méthodologiques originaux, les " codes pathologie » et "l'indice pathologique ». Une trentaine d'individus par station et par campagne sont capturés par pêche électrique à un rythme hebdomadaire durant la période estivale. Ils appartiennent essentiellement aux espèces suivantes : chevaine (Squalius cephalus) et gardon (Rutilus rutilus), puis perche fluviatile (Perca fluviatilis), barbeau fluviatile (Barbus barbus) et goujon (Gobio gobio). Bien qu'un écart moyen de température de l'eau de l'ordre de $1,3^{\circ} \mathrm{C}$ soit observé pour la température tendancielle lors de nos campagnes d'échantillonnage au cours de la saison estivale (fin juin - début septembre) entre la station aval échauffée et la station amont $\left(\min 0,4^{\circ} \mathrm{C}, \max 2,3^{\circ} \mathrm{C}\right)$, l'étude ne met pas en évidence de différence significative des indices de condition moyens ( $\mathrm{lcm}$ ) ainsi que des prévalences des lésions externes moyennes des poissons entre l'amont et l'aval après mélange. En revanche, l'effet combiné température et débit pourrait expliquer la variation interannuelle des prévalences de lésions observées au cours de l'étude. Le maximum de lésions est observé lors des années chaudes et à faible débit (2009) alors que les années froides et à fort débit sont caractérisées par des prévalences de lésions plus faibles (2013). Au final, aucun des effets constatés, directs ou indirects, n'est spécifique de l'action de la seule augmentation de température et ne démontre pas de manière significative une action de celle-ci sur les différents indices utilisés et, donc, sur l'état de santé des poissons. Le débit est également susceptible de représenter un facteur de stress majeur pour l'ichtyofaune et d'agir sur l'intégrité des individus. En revanche, la qualité physico-chimique standard et toxique ne semble pas être un facteur discriminant sur le Rhône. Les lésions observées dans le cadre de cette étude sont 
plus le fait d'atteintes pathologiques mineures, essentiellement provoquées par des bactéries opportunistes, que de problèmes toxicologiques. Dans le cadre de l'effet de plus en plus prégnant du changement climatique global sur les hydrosystèmes continentaux, une réflexion sur la prise en compte et le suivi des effets potentiels du réchauffement engendré sur le développement des maladies des poissons et sur le comportement des espèces résidentes et migratrices semble nécessaire, d'autant plus que d'autres descripteurs (hématologiques et histologiques) sont également susceptibles d'être intégrés dans ces outils de diagnostic et de surveillance de l'état de santé des populations piscicoles.

Mots-clés - pathologie pisciaire, température, poissons, fleuve Rhône

\begin{abstract}
Health monitoring of fish populations of the Rhône River has been set up each summer since 2005 upstream and downstream of the Tricastin nuclear plant as part of the preventive survey in the case of a thermal hazard impacting electricity production. It is based on relevant and validated biological descriptors (condition index, parasitism) and two original methodological tools called «pathology codes » and «pathology index ». Thirty individuals per station per campaign are caught by electric fishing weekly during summer. They mainly belong to the following species: chub (Squalius cephalus) and roach (Rutilus rutilus) and then perch (Perca fluviatilis), barbel (Barbus barbus) and gudgeon (Gobio gobio). The lack of significant difference in average condition indices $(\mathrm{Icm})$ and average prevalences of external lesions of fish between the upstream and downstream after mixing can be explained by a difference in temperature not quite marked between the two stations $\left(\Delta \mathrm{T}=1.3^{\circ} \mathrm{C}\right.$ on average, $2.3^{\circ} \mathrm{C}$ maximum). However, the combined effect of temperature and discharge could explain the annual variation in the prevalence of lesions observed during the study. The maximum of lesions is observed in warm years with low discharge (2009) while the cold years with high discharge are characterized by lower prevalence of lesions (2013). Finally, none of these effects, direct or indirect, is specific of the temperature rise only and does show a significant action of those on the various indices used and, therefore, on the fish health. Discharge can also be a major stress factor for the fish fauna and act on the integrity of individuals. On the opposite, the standard physicochemical and toxic quality does not seem to be a discriminating factor on the Rhône River. The lesions observed in this study are due to minor pathological reaches, essentially caused by opportunistic bacteria, rather than toxicological ones. As part of the study of more and more significant impacts of global climate change on hydrosystems, a reflection on the recognition and monitoring of the potential effects of global warming led to the development of fish diseases and to the behavior of the resident and migratory species seems necessary, particularly as other descriptors (hematological and histological) are also likely to be integrated into these diagnostic and monitoring tools of the health of fish populations.
\end{abstract}

Key words - fish patology, Temperature, fish communities, Rhône River

\section{INTRODUCTION}

D'après la bibliographie (Travade, 1975 ; Girard, 1998, 2009), la température jouerait un rôle majeur dans l'apparition, le développement et la régulation des agents pathogènes (microorganismes et micropolluants) et des maladies des poissons, la majorité des effets constatés étant directement corrélée avec l'augmentation de la température. Elle agirait effectivement 
sur tous les composants du milieu et à tous les niveaux des chaînes trophiques, mais aucun de ses effets sur la santé des poissons, directs ou indirects, n'est spécifique de son action. En cas de températures trop élevées par rapport au préférendum thermique de chaque espèce, les répercussions sur les poissons pourraient être de deux types:

- directes et immédiates, caractérisées par l'apparition de lésions anatomo-morphologiques, de perturbations physiologiques diverses (respiration, nutrition par disparition de nourriture spécifique, reproduction) et, surtout, l'instauration d'un état de stress et l'apparition de la maladie ; indirectes et différées, en relation avec la présence à faibles doses dans le milieu de substances toxiques aux effets insidieux. À terme, outre l'affaiblissement et l'état de misère physiologique qui caractériseraient les individus ayant survécu, c'est la pérennité des espèces qui pourrait être mise en jeu.

Dans le cadre du «Programme de surveillance en situations exceptionnelles » des Centres Nucléaires de Production d'Électricité (CNPE) dans le cas d'un aléa climatique impactant la production (ex arrêté « Situation climatique exceptionnelle » du 11 Juin 2004), une surveillance sanitaire des populations piscicoles a été mise en place chaque été depuis 2005 en amont et en aval de deux CNPE : celui du Tricastin de façon systématique préventive et celui du Bugey de façon optionnelle en cas de canicule avérée. Une telle intervention est également proposée dans le cadre du suivi hydrobiologique renforcé du CNPE de SaintAlban, mais elle n'y a pour l'instant pas été réalisée.

Ces études diffèrent de celles entreprises antérieurement en proposant une approche globaliste et beaucoup plus généraliste. Elles s'appuient en outre sur des descripteurs biologiques pertinents et validés (indice de condition, parasitisme) et sur deux outils méthodologiques originaux, les « codes pathologie » et «l'indice pathologique ».

La présente étude s'intéresse à la chronique des mesures effectuées sur le CNPE du Tricastin depuis l'été 2008, chaque campagne s'inscrivant dans une suite logique et cohérente par rapport à celle réalisée l'année précédente en utilisant un protocole et une méthodologie similaires.

\section{MATÉRIEL ET MÉTHODES}

\subsection{Stations et protocole d'échantillonnage}

Le suivi est réalisé sur deux stations, respectivement en amont et en aval du CNPE du Tricastin.

La station de référence amont est située en aval de Viviers, à l'entrée du défilé de Donzère, centrée sur le pk 167, essentiellement en rive droite. La station aval est située dans la partie terminale du canal de Donzère-Mondragon, en amont de la restitution avec le Rhône court-circuité, centrée sur le pk 200, sur les deux rives. Cette dernière peut être considérée comme étant une station aval éloignée après mélange car le rejet d'eau réchauffée 
est situé sur le Rhône dans le canal de dérivation de l'aménagement CNR de Donzère-Mondragon au pk 184,5, en amont de l'écluse de Bollène (Fig. 1).

Des poissons de différentes espèces sont prélevés par pêche électrique selon un rythme hebdomadaire entre fin juin et début septembre, afin de constituer des échantillons représentatifs sur lesquels différents examens et analyses sont réalisés. Les pêches sont effectuées en bateau à l'aide d'un matériel EFKO FEG 8000.

Une trentaine d'individus est pêchée par station et par campagne. Ils appartiennent essentiellement aux espèces suivantes : chevaine (Squalius cephalus) et gardon (Rutilus rutilus), puis perche fluviatile (Perca fluviatilis), barbeau fluviatile (Barbus barbus) et goujon (Gobio gobio). Le chevaine est l'espèce de poisson dominante du secteur tandis que les autres taxons font partie du fond ichtyologique régulier de ce secteur du Rhône (Carrel et al., 2013).

\subsection{Examens et analyses sanitaires}

Les opérations suivantes sont entreprises in situ sur chaque poisson fraîchement pêché et préalablement sacrifié par surdosage d'anesthésique (2-PHENOXY-ETHANOL : 1,0 ml/litre d'eau) :

- identification de l'espèce ;

- mesures biométriques : longueur totale ( $L$ en millimètres), poids ( $P$ en grammes) et calcul de l'indice de condition (IC) (NB1) ;

- examens macroscopiques externes (NB2) et internes destinés à noter toute altération ou lésion éventuelle (selon la grille «Codes pathologie »
(NB3) ci-après), avec calcul des indices pathologiques relatifs à chaque type de lésion et globaux (IpG) (NB4) pour chaque station ;

- examens parasitologiques externes et internes (œil nu et loupe optique) destinés à apprécier l'ambiance parasitaire globale :

- cutanéo-branchiale : Crustacés (Argulus, Lernées) principalement, mais aussi « Points blancs » (= Ichtyophtirius multifiliis) et Myxosporidies (Myxobolus sp., Henneguya),

- interne : Acanthocéphales, Cestodes, Nématodes

- examen détaillé des branchies à la loupe optique (X 8) ou binoculaire (X 20) selon les cas.

NB1 : L'indice de condition (Ic) est une relation mathématique entre le poids $(P)$ et la taille $(L)$ des animaux qui s'exprime selon la formule : Ic $=$ $\left[P / L^{3}\right]^{\star} 10^{-5}$, où $P=$ masse en grammes et $L^{3}=$ longueur totale exprimée en millimètres au cube. Cet indice offre un intérêt certain pour évaluer la condition des poissons en fonction de la qualité et du degré de trophie du milieu dans lequel ils évoluent (Bogé et al., 1991).

NB2 : Les anomalies des poissons sont généralement l'expression d'altérations physiologiques ou biochimiques provoquées par la présence dans l'environnement de substances toxiques ou de bioagresseurs. Ces altérations morphologiques permettent d'évaluer les effets d'expositions de longues durées à de faibles niveaux de contamination, en particulier les lésions tissulaires, représentées par des érosions (cutanées ou branchiales), des nécroses, des ulcères (du tégument), des cata- 

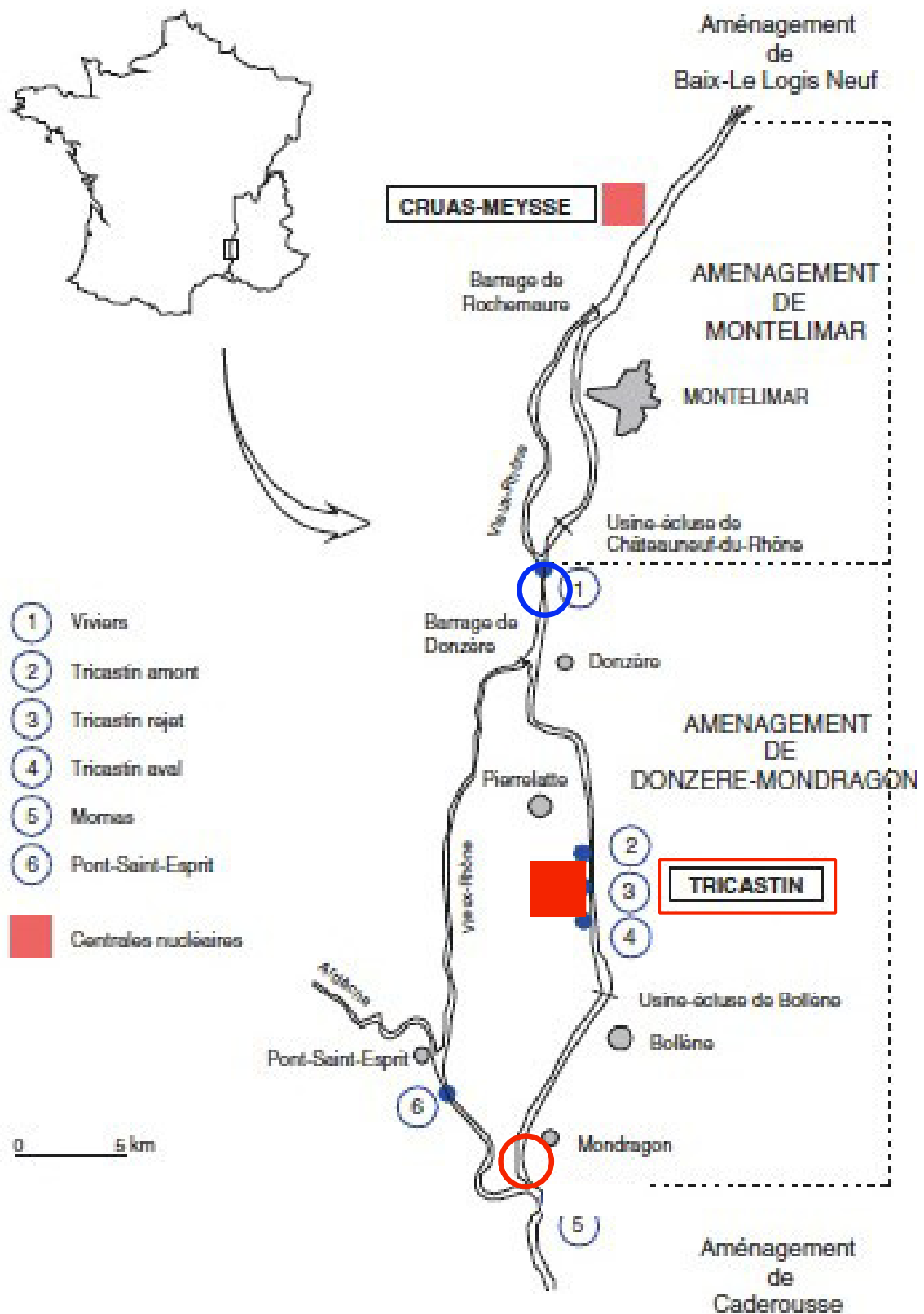

(1) Viviars

(2) Tricastin amort

(3) Tricastin rejot

(4) Tricastin aval

(5) Mornas

(6) Pont-Saint-Esprit

Controles nuelsiaires

Fig. 1. Le Rhône à Tricastin et localisation des stations d'échantillonnage. Fond de carte G. Carrel (Irstea Aix-en-Provence).

Fig. 1. The Rhône river at Tricastin and location of the sampling stations. Base map from G. Carrel (Irstea Aix-en-Provence). 
Grille d'interprétation des IpG :

\begin{tabular}{|l|ccccc|}
\hline $\begin{array}{l}\text { Classes } \\
\text { de qualité }\end{array}$ & $0 \Rightarrow 0,04$ & $\mathbf{0 , 0 5} \Rightarrow \mathbf{0 , 2 0}$ & $\mathbf{0 , 2 1} \Rightarrow \mathbf{0 , 8 0}$ & $\mathbf{0 , 8 1} \Rightarrow \mathbf{1 , 4 0}$ & $\mathbf{1 , 4 1 \Rightarrow 4 , 0 0}$ \\
\cline { 1 - 1 } Etat sanitaire & Excellent & Bon & Précaire & Dégradé & Mauvais \\
\hline
\end{tabular}

ractes, des hyperplasies, des déformations (squelette ou colonne vertébrale) et, enfin, les tumeurs (Baumann, 1996 ; Nounou, 1980 et 1985).

Basé sur l'observation des anomalies externes des poissons, l'outil «Codes pathologie » permet donc de renseigner sur l'état de santé des populations ou peuplements piscicoles ainsi que sur l'aspect, la nature et les étiologies possibles (= orientation de diagnostic) des différentes lésions constatées (Girard, 1998 ; Girard \& Elie, 2007a, 2007b ; Elie \& Girard, 2014).

NB3 : Les lésions anatomo-morphologiques sont décrites par un code à 3 lettres dont les deux premières font référence à la nature même des lésions observées (exemple : ulcère hémorragique $=$ «UH $》)$ et la $3^{\circ}$ à leur localisation (exemple : flanc $=« F »)$.

NB4 : L'indice «pathologique » (Ip) est une relation mathématique entre la prévalence de poissons présentant des lésions externes et l'intensité de chaque lésion observée.

Cet indice, de nature quantitative et qualitative, permet d'établir des comparaisons intra- et interspécifiques, mais également inter-stationnelles et spatio-temporelles.

Pour une lésion donnée, l'indice pathologie (Ip) s'exprime par la formule : $\mathrm{IP}=\mathrm{P} \times \mathrm{Q}$, où :

$P$ représente le $\%$ de poissons présentant des lésions externes, $Q$ représente la sévérité lésionnelle.
Pour une population donnée, l'indice pathologie global (IpG) additionne les IP relatifs à chaque lésion observée et s'exprime ainsi : IpG $=\Sigma I P$.

\subsection{Modalités d'interprétation}

Treize types de données sont renseignés selon le Tableau I.

\section{Codes " Pathologie" : grille de description}

Différents codes pathologiques sont relevés avec une grille de description (cf. Tab. II).

\section{RESULTATS}

\subsection{Hydrologie et température de la période d'étude}

L'hydrologie annuelle fait ressortir trois années normales $(2008,2010$, 2012), deux années déficitaires (2009 et, surtout, 2011) et une année très excédentaire (2013) (Fig. 2). Les événements hydrologiques les plus marquants vis-à-vis de cette étude et de sa localisation temporelle (période estivale post reproduction) sont le déficit printanier 2011 et, inversement, l'excédent printanier 2013.

Du point de vue thermique, l'année 2011 est particulièrement chaude en 
Tableau I. Données renseignées à chaque station lors de chaque campagne.

Table I. Data recorded at each station during each sampling campaign.

\begin{tabular}{|c|c|}
\hline Colonne 1 & espèce (ESP) \\
\hline Colonne 2 & longueur totale ( $\mathrm{Lmm})$ exprimée en millimètres \\
\hline Colonne 3 & poids $(\mathrm{Pg})$ exprimé en grammes \\
\hline Colonne 4 & indice de condition (IC) \\
\hline Colonne 5 & lésions macroscopiques externes (Cp ext) \\
\hline Colonne 6 & nombre de lésions macroscopiques externes observées (N) \\
\hline Colonne 7 & microparasitisme cutanéo-branchial $(\mu \mathrm{Pa})$ \\
\hline Colonne 8 & $\begin{array}{l}\text { abondance parasitaire }(\mathrm{Ab}) \text { exprimée en termes de classes de qualité } \\
\text { conformément à la grille «Codes pathologie » }\end{array}$ \\
\hline Colonne 9 & lésions macroscopiques internes (Cp int) \\
\hline Colonne 10 & nombre de lésions macroscopiques internes observées $(\mathrm{N})$ \\
\hline Colonne 11 & $\begin{array}{l}\text { parasites macroscopiques (PaTD) observés dans le tube digestif (intestin } \\
+ \text { estomac) }\end{array}$ \\
\hline Colonne 12 & $\begin{array}{l}\text { abondance parasitaire }(\mathrm{Ab}) \text { exprimée en termes de classes de qualité } \\
\text { conformément à la grille « Codes pathologie » }\end{array}$ \\
\hline Colonne 13 & état des branchies. \\
\hline
\end{tabular}

raison essentiellement d'un réchauffement printanier précoce et du prolongement de la douceur tard en automne (Fig. 2). Les événements thermiques les plus marquants sont la " chaleur » du printemps 2011 opposée à la fraîcheur des printemps 2012 et 2013 . Le delta T engendré par le rejet, après mélange (pk 192 en aval de l'écluse de Bollène), est en moyenne de $1,5^{\circ} \mathrm{C}$ sur l'ensemble de la période d'étude 2008-2013 $\left(\min =0{ }^{\circ} \mathrm{C} ; \max =4,7^{\circ} \mathrm{C}\right.$ en octobre 2009 et en novembre 2011 ; méd $=1,3^{\circ} \mathrm{C}$ ). Au niveau de notre station de pêche aval (pk 200), on peut donc considérer que le rejet a totalement diffusé dans la masse d'eau tout en se diluant.

Pour chaque campagne, les débits et les températures moyens des 7 jours ayant précédé l'échantillonnage ont été calculés, i.e. débits et températures tendanciels (Fig. 3 ).
Les résutats montrent une variabilité des situations hydrologiques interannuelles (débit moyen tendanciel de $784 \mathrm{~m}^{3} / \mathrm{s}$ en 2009 vs $1421 \mathrm{~m}^{3} / \mathrm{s}$ en 2008). Une tendance générale à la baisse du débit au cours de chaque étude annuelle est notée, hormis en 2011 où l'hydrologie a été variable mais toujours inférieure à $1000 \mathrm{~m}^{3} / \mathrm{s}$. Concernant la température, le delta $T$ estival tendanciel moyen lors de l'ensemble des campagnes (périodes annuelles fin juin - début septembre) entre l'amont et l'aval du CNPE après mélange est de $1,3{ }^{\circ} \mathrm{C}\left(\min =0,4^{\circ} \mathrm{C}\right.$ début juillet 2008 ; $\max =2,3^{\circ} \mathrm{C}$ en juillet 2011 ; méd $=1,3^{\circ} \mathrm{C}$ ). La tendance à l'accroissement de la température en cours de saison pour chaque étude annuelle est moins nette que celle (inverse) des débits : ceci est net en 2012 et 2013 avec le réchauffement du fleuve en juillet puis la stabilité de la 
Tableau II. Grille de description des Codes pathologie.

Table II. Description grid of pathology codes.

\begin{tabular}{|c|c|c|c|}
\hline \multicolumn{4}{|c|}{$\begin{array}{c}\text { Grille de codification des anomalies anatomo-morphologiques externes et des } \\
\text { ectoparasites des poissons visibles à l'œil nu }\end{array}$} \\
\hline $\begin{array}{l}\text { Altérations anatomo- } \\
\text { morphologiques }\end{array}$ & AA & Localisations anatomiques & Code \\
\hline Absence d'organes & $\mathrm{AO}$ & Tout le corps & $\mathrm{C}$ \\
\hline Bulles de gaz & $B G$ & Tête & $T$ \\
\hline $\begin{array}{l}\text { Déformations, difformités et } \\
\text { malformations }\end{array}$ & $A D$ & Mâchoire & M \\
\hline Erosions & ER & Bouche & $\mathrm{G}$ \\
\hline Nécroses & $\mathrm{NE}$ & Barbillon & $\mathrm{J}$ \\
\hline Hypersécrétion de mucus & $\mathrm{HM}$ & Narine & $\mathrm{N}$ \\
\hline Hypertrophie et hyperplasie & $\mathrm{HY}$ & CEil & $\bar{Y}$ \\
\hline $\begin{array}{l}\text { Lésions hémorragiques et } \\
\text { congestives }\end{array}$ & HE & $\begin{array}{l}\text { Opercule (fente branchiale chez } \\
\text { l'anguille) }\end{array}$ & $\mathrm{O}$ \\
\hline Maigreur & AM & Branchie (sauf pour l'anguille) & B \\
\hline $\begin{array}{l}\text { Plaies et lésions anciennes } \\
\text { (cicatrices) }\end{array}$ & PL & $\begin{array}{l}\text { Dos (= face pigmentée ou } \\
\text { zénithale) }\end{array}$ & D \\
\hline $\begin{array}{l}\text { Tumeurs, kystes, nodules et autres } \\
\text { grosseurs }\end{array}$ & $\mathrm{TG}$ & Colonne vertébrale & V \\
\hline $\begin{array}{l}\text { Ulcères (hémorragiques) et } \\
\text { ulcérations }\end{array}$ & $\mathrm{UH}$ & Flanc & $\mathrm{F}$ \\
\hline Etat pathologique multiforme & $\mathrm{ZO}$ & Ligne latérale & $\mathrm{L}$ \\
\hline Altérations de la couleur & AC & Ecaille & $E$ \\
\hline Opacité & OP & $\begin{array}{l}\text { Abdomen (= face aveugle ou } \\
\text { nadirale) }\end{array}$ & A \\
\hline Coloration sombre (mélanose) & CS & Orifice urogénital (anus) & $\mathrm{U}$ \\
\hline $\begin{array}{l}\text { Coloration terne ou pâle, } \\
\text { dépigmentation }\end{array}$ & CT & Nageoire dorsale & $\mathrm{H}$ \\
\hline Parasitisme & PA & Nageoire pectorale & $P$ \\
\hline Points blancs & PB & Nageoire pelvienne & $\mathrm{R}$ \\
\hline Points noirs & $\mathrm{PN}$ & Nageoire anale & $S$ \\
\hline Crustacés & PC & Nageoire caudale & $Q$ \\
\hline Hirudinés (sangsues) & $\mathrm{PH}$ & $\begin{array}{l}\text { Nageoire adipeuse (salmonidés et } \\
\text { ictaluridés) }\end{array}$ & $\mathrm{J}$ \\
\hline Champignons & PM & Pédoncule caudal & $\mathrm{K}$ \\
\hline
\end{tabular}


Tableau II. Suite.

Table II. Continued.

\begin{tabular}{|c|c|c|c|}
\hline \multicolumn{4}{|c|}{ Grille de quantification des anomalies et du parasitisme externes } \\
\hline $\begin{array}{l}\text { Nombre de lésions }(\mathrm{N}) \text { et/ou } \\
\text { abondance parasitaire (Ab) }\end{array}$ & Code & $\begin{array}{l}\text { Taux }(\%) \text { de recouvrement } \\
\text { corporel }\left(\mathrm{S}^{2}\right)\end{array}$ & Code \\
\hline $\mathrm{N}=0$ et/ou absence $(\mathrm{Ab})$ & 0 & $S^{2}=0 \%$ & 0 \\
\hline $\mathrm{N}<3$ et/ou abondance $(\mathrm{Ab})$ faible & 1 & $\mathrm{~S}^{2}<5 \%$ & 1 \\
\hline $\mathrm{N}=4-6$ et/ou abondance $(\mathrm{Ab})$ moyenne & 2 & $S^{2}=5-10 \%$ & 2 \\
\hline $\mathrm{N}=7-10$ et/ou abondance $(\mathrm{Ab})$ forte & 3 & $S^{2}=10-20 \%$ & 3 \\
\hline $\mathrm{N}=>10$ et/ou abondance $(\mathrm{Ab})$ très forte & 4 & $S^{2}=>20 \%$ & 4 \\
\hline
\end{tabular}

température en août. De la même manière que pour l'hydrologie, l'année 2008 s'oppose à 2009 (température tendancielle amont moyenne de $19,7^{\circ} \mathrm{C}$ vs $22,5^{\circ} \mathrm{C}$, pour des deltas $\mathrm{T}$ de $1,1^{\circ} \mathrm{C}$ vs $1,6^{\circ} \mathrm{C}$ ).

La figure 4 résume quelques statistiques des deux types de données thermiques, journalières d'une part $(A)$, estivales tendancielles 7 jours d'autre part (B).

\subsection{Résultats piscicoles}

\subsubsection{Effectifs}

De 9 à 13 campagnes sont réalisées selon les années. Les résultats sont détaillés dans le tableau III. Plus de 5300 poissons appartenant à 18 espèces piscicoles ont été analysés, respectivement 2709 en amont et 2615 en aval. Comme défini dans la méthodologie, ils appartiennent essentiellement à 5 espèces, les individus des autres espèces ayant été conservés « accidentellement ": chevaine
( $51,6 \%$ des individus), gardon (27,4\%), perche fluviatile $(6,1 \%)$, barbeau fluviatile $(6,0 \%)$ et goujon $(5,0 \%)$.

\subsubsection{Indices de condition}

Ils ont été calculés pour les deux principales espèces, le chevaine et le gardon.

Au cours de l'étude, de 2008 à 2013 (70 dates d'échantillonnage), l'indice de condition moyen $(\mathrm{Icm})$ des chevaines n'est pas significativement différent à l'amont et à l'aval du CNPE (respectivement 0,943 et 0,$942 ; \mu<\mu_{95 \%}$ soit $0,009<1,67)$. Aucun « effet CNPE 》 n'est détecté. En revanche, lors d'une comparaison interannuelle des $\mathrm{Icm}$ entre les deux stations (Fig. 5), des différences significatives apparaissent (test de Mann Whitney ; Uamont $<U(0,6))$. Cependant, aucune règle ne peut être établie : au cours des 6 années, à 3 reprises les $\mathrm{Icm}$ amont sont plus élevés que ceux de l'aval (2008, 2009 et 2013) alors que c'est l'inverse lors des 3 autres années (2010, 2011 et 2012). 

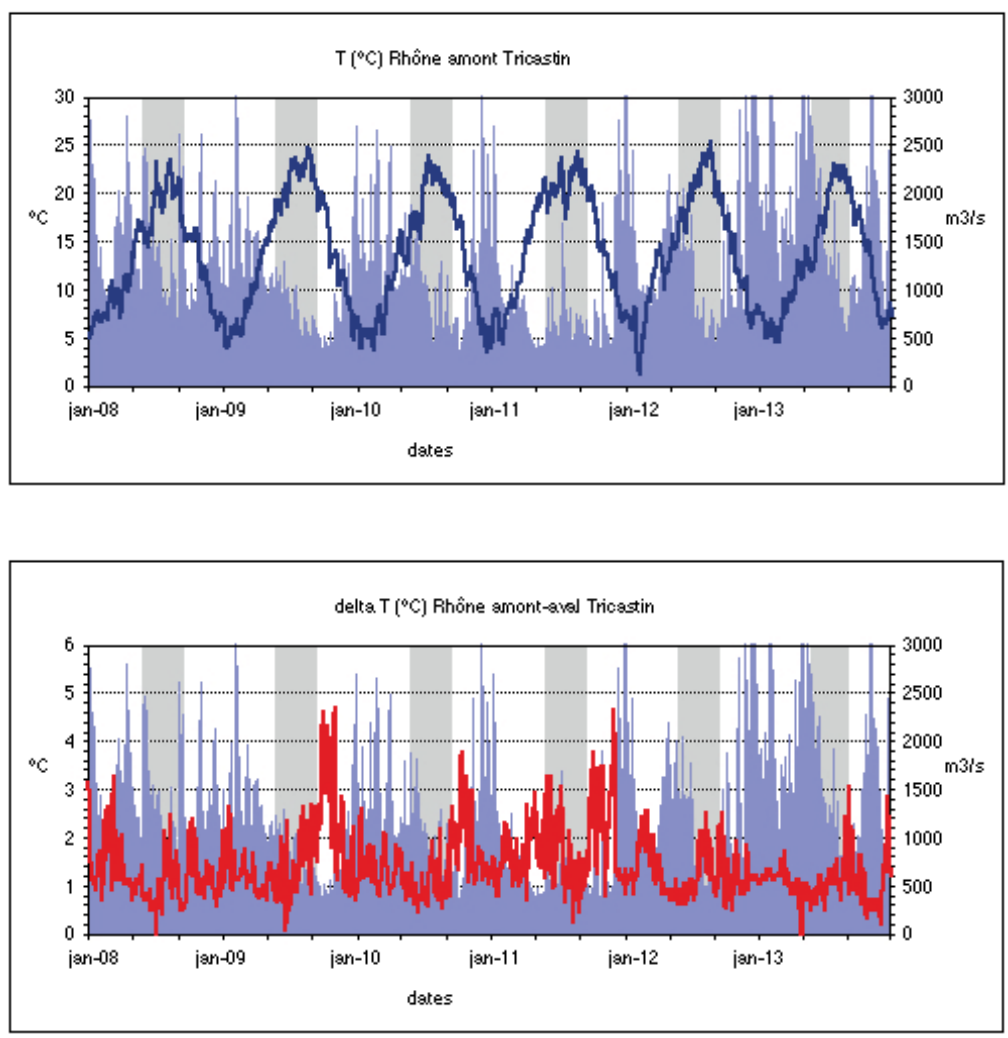

Fig. 2. Débit et température moyens journaliers du Rhône entre 2008 et 2013 et localisation des périodes annuelles de suivi (grisé en arrière plan). Débit à la station de Viviers, température en amont et aval immédiats du CNPE. La température est exprimée selon deux points de vue et le débit est figuré en arrière plan. Données G. Carrel (Irstea Aix-en-Provence).

Fig. 2. Average daily discharge and temperature of the Rhône river between 2008 and 2013 and location of the annual monitoring periods (gray background). Discharge at Viviers station, temperature upstream and immediatly downstream of the NPP. The temperature is expressed in two point of view and the discharge is drawn in the background. Data from G. Carrel (Irstea Aix-enProvence).

Avec un peu moins de données que pour le chevaine (respectivement 43 et 46 dates à l'amont et à l'aval) dû à des effectifs parfois trop faibles $(n<$ 10 ; en particulier en 2010 et 2011), l'Icm des gardons n'est pas non plus significativement différent entre les deux stations (respectivement 0,958 et 0,982 à l'amont et à l'aval ; $\mu<\mu_{95 \%}$ soit $0,183<1,68)$.
Malgré un delta moyen de $1,3^{\circ} \mathrm{C}$ en faveur de la station aval par rapport à la station amont (respectivement $22,6^{\circ} \mathrm{C}$ et $21,3^{\circ} \mathrm{C}$ au cours des 6 années), les chevaines et les gardons ont des rapports moyens longueur/masse identiques entre les deux stations.

Aucune tendance réellement significative n'est décelée le long de la chronique d'étude concernant les $\mathrm{Icm}$ 


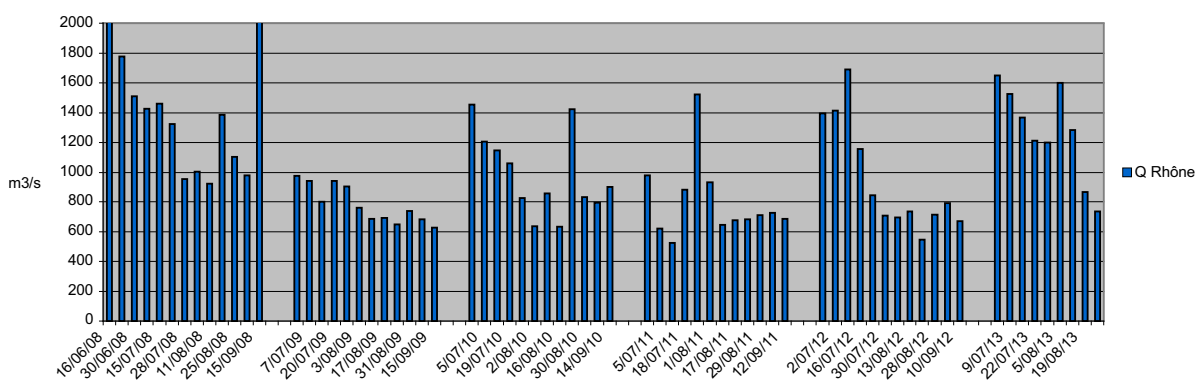

dates

Températures tendancielles 2008-2013

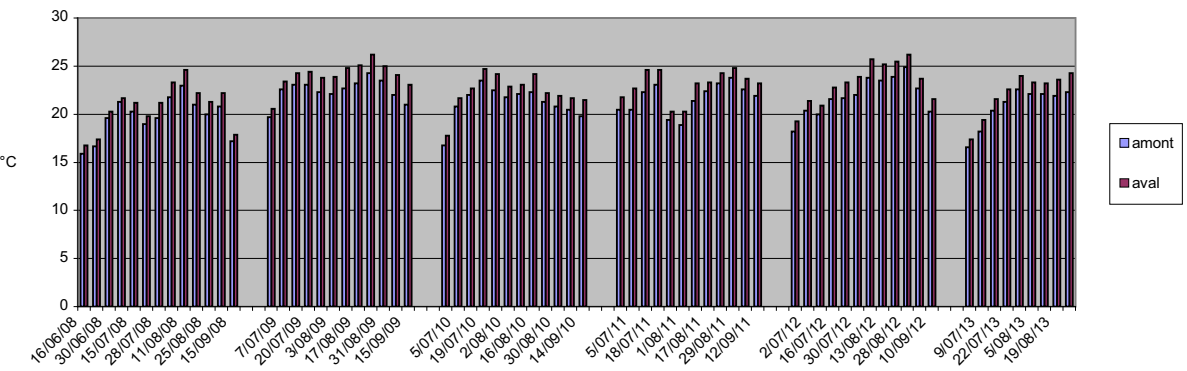

dates

Fig. 3. Débit et température amont-aval tendanciels 7 jours pour chaque campagne d'échantillonnage.

Fig. 3. Upstream-downstream discharge and temperature of the 7 days before each sampling campaign.

annuels des chevaines, que ce soit à l'amont ou à l'aval (respectivement $R^{2}=0,390$ et $R^{2}=0,217$; Figure 5).

Pour le gardon, les faibles effectifs, en particulier en 2010 et $2011(n<10)$, n'ont pas permis de calculer la tendance le long de la chronique d'étude.

L'indice de condition du chevaine a tendance à diminuer en cours de saison à la station amont. II est beaucoup plus fluctuant à l'aval. À la station amont, la baisse est significative lors des 4 premières années (de 2008 à 2011 avec $R^{2}>0,5$ ) alors qu'elle ne l'est pas lors des 2 dernières (2012 et 2013 avec $\left.\mathrm{R}^{2}<0,5\right)$ (Fig. 6). À la station aval, seul la baisse de l'année 2009 est tout juste significative $\left(R^{2}=\right.$ $0,500)$.

\subsubsection{Prévalences des lésions}

Sur les quelques 5300 poissons capturés et analysés, près de $30 \%$ d'entre eux présentent des lésions externes (respectivement $29,1 \%$ et $29,4 \%$ en amont et en aval sur la 


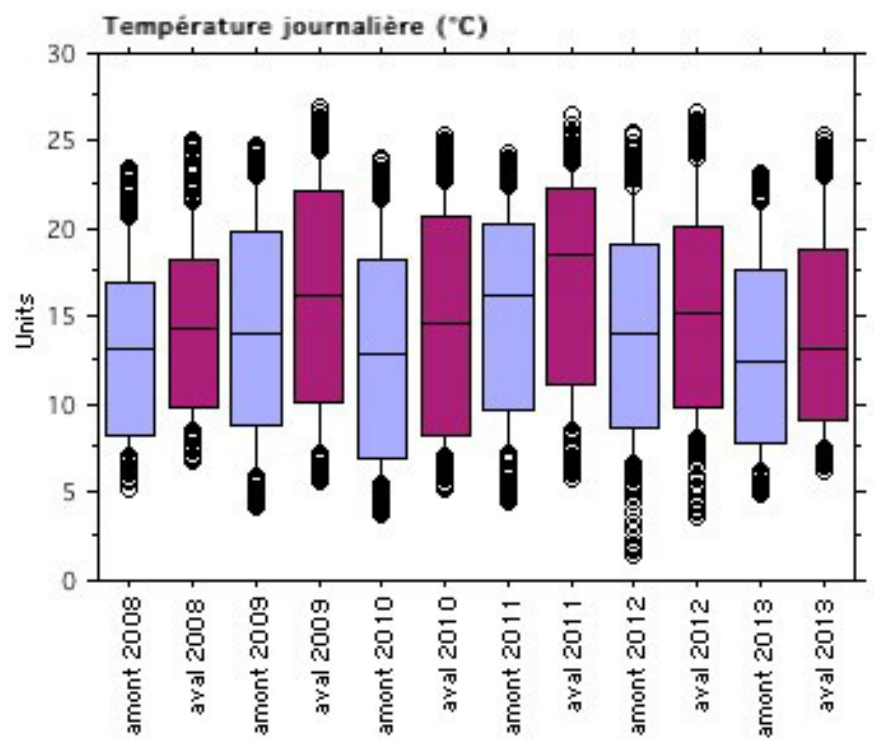

Fig. 4. Distribution inter-annuelle stationnelle des données thermiques. A - Température moyenne journalière. B - Température estivale tendancielle 7 jours. Distribution selon le principe des « boites et moustaches " des valeurs de température $\left({ }^{\circ} \mathrm{C}\right)$. Chaque boite est délimitée par les 1er et $3^{\mathrm{e}}$ quartiles. La ligne du milieu représente la médiane. Les points représentent les valeurs extrêmes situées audelà des $1^{\mathrm{er}}$ et $9^{\mathrm{e}}$ déciles (« moustaches ").

Fig. 4. Distribution between years and between stations of thermal data. A - Average daily temperature. B - Summer temperature of the 7 days before sampling. Distribution according to the principle of "box and whiskers plot" of the temperature values $\left({ }^{\circ} \mathrm{C}\right)$. Each box is bounded by the first and third quartiles. The middle line represents the median. The dots represent extreme values located beyond the first and ninth deciles ("whiskers").

globalité de la chronique). Cette différence n'est cependant pas significative $\left(\mu<\mu_{95 \%}\right.$ soit $\left.0,018<1,645\right)$. Aucun « effet CNPE " n'est détecté concernant la prévalence des lésions externes entre les deux stations.

Aucune différence significative est notée pour une même année entre les deux stations (test de Mann Whitney ; Uam > U(0,6)) (Fig. 7). II est alors possible de regrouper les données annuellement pour une comparaison interannuelle (Fig. 8).

L'année 2013 est celle où les poissons apparaissent les plus sains (moins de $15 \%$ de poissons avec lésions) tandis que près de $50 \%$ des poissons sont atteints de lésions en 2009 , année à faible hydrologie et température la plus élevée. De plus, une baisse significative des prévalences apparaît au fil de l'étude $\left(R^{2}=0,552\right)$.

Une nette chute des prévalences est enregistrée à partir de 2010 par rapport aux années 2008 et 2009. L'année 2013 présente de nombreuses prévalences parmi les plus basses (hydrologie plutôt élevée, seconde température tendancielle moyenne la plus basse).

Parmi les nombreux types de lésions pathologiques et parasitaires rencontrés (cf. Tab. II), seulement 5 
可弯

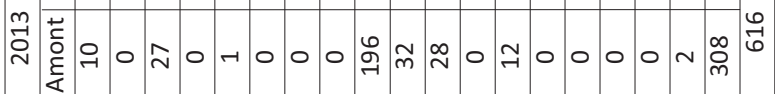

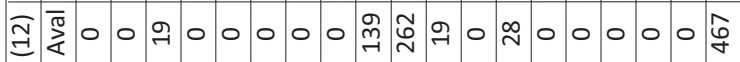

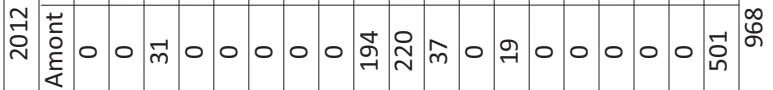

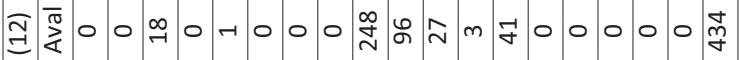
突

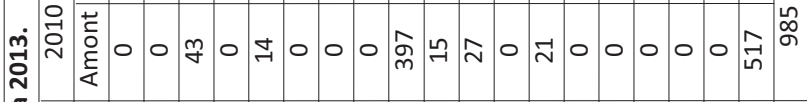

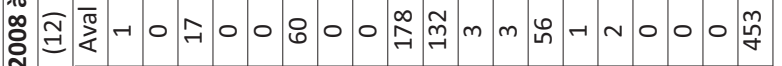

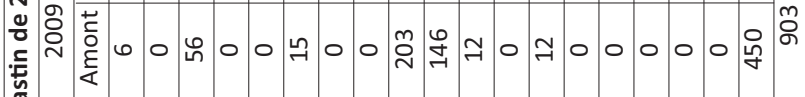

\&

중

कृ

즐

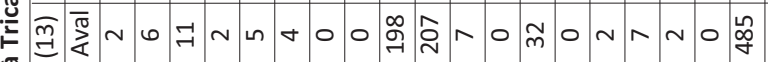

⿹气巳 


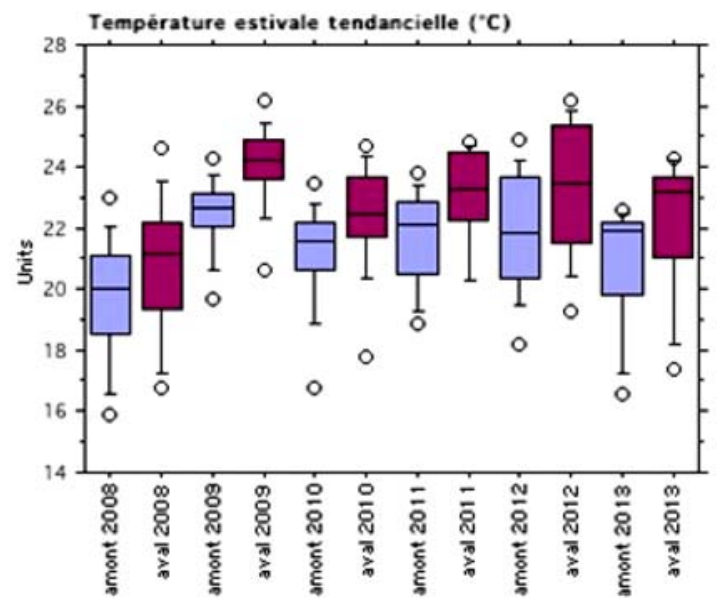

Icm CHE amont et aval (2008-2013)

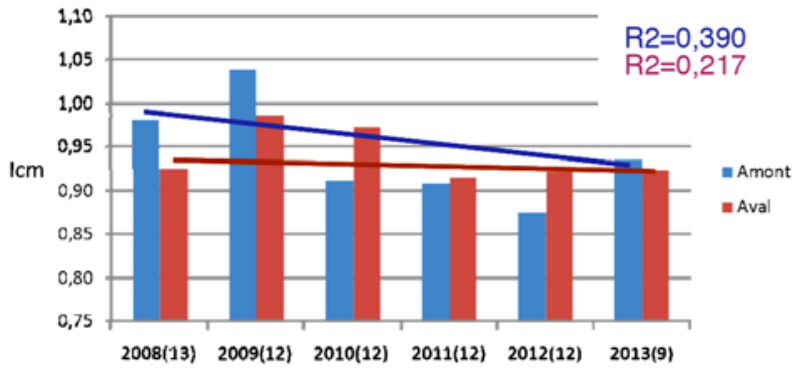

Fig. 5. Indices de condition (Icm) annuels du chevaine à l'amont et à l'aval du CNPE.

Fig. 5. Annual condition index $(\mathrm{Icm})$ of chub upstream and downstream of the NPP.

d'entre eux présentent une prévalence $>1 \%$ au total, regroupant respectivement en amont et en aval $97 \%$ et $96 \%$ des poissons atteints. Il s'agit des érosions (code $E R=42 \%$ des individus avec lésions en amont et $54 \%$ en aval), des nécroses (NE), des hémorragies (HE) et des points noirs (PN) environ $15 \%$ en moyenne de poissons atteints quelle que soit la station - ainsi que des ulcères hémorragiques $(\mathrm{UH}$ : légèrement $>1 \%$ à chacune des deux stations). Si les érosions (ER) semblent significativement plus nombreuses à la station aval (excepté en
2013), la distribution est plus variable pour les autres types de lésions selon les années (Fig. 9).

Par ailleurs, 78,3\% des lésions - et ce quelle que soit leur nature - sont localisées au niveau de la nageoire caudale (Q) et 79,9\% des lésions sont d'intensité faible (de niveau 1 ).

Sans être réellement spécifique d'une affection particulière, la nageoire caudale est généralement la cible privilégiée de nombreuses bactéries pathogènes opportunistes (Aeromonas sp., Pseudomonas sp. Flavobacterium sp.) et de plusieurs ectoparasites parmi 

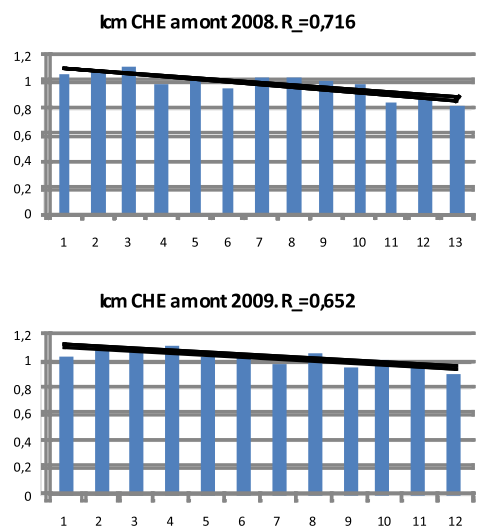

lcm CHE amont 2010.R_=0,775

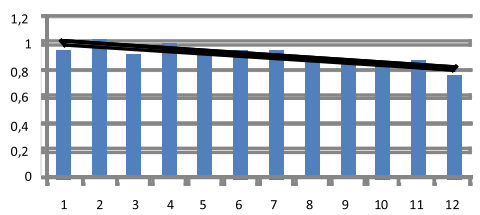

$\mathrm{km}$ CHE amont 2011. $R_{-}=0,682$

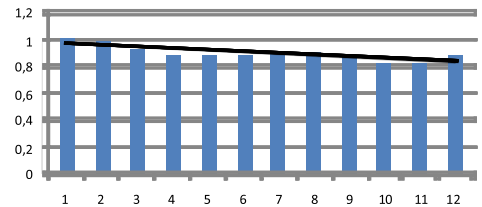

Icm CHE amont 2012. $R_{-}=0,323$

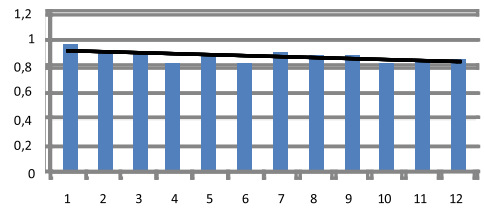

Iom CHE amont 2013. $\mathrm{R}=\mathbf{0 , 1 5 0}$

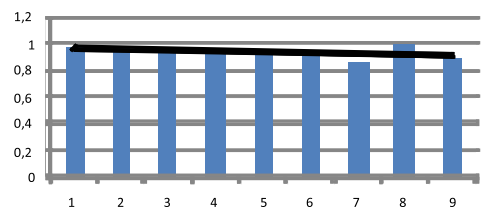

Ian CHE aval2008. $R_{-}=0,187$

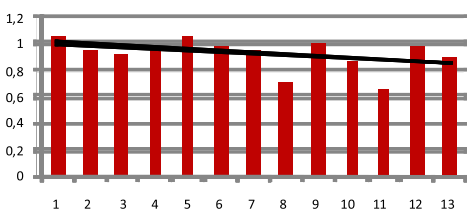

Icm CHE aval2009. $R=0,500$

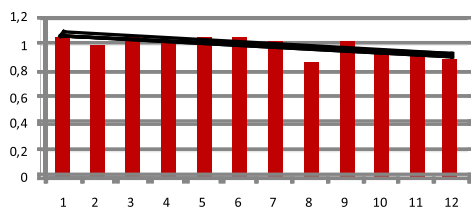

Icm CHE aval2010. $R=0,263$

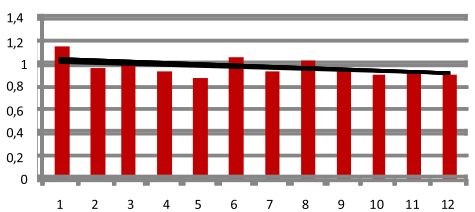

Iom $\mathrm{CHE}$ aval 2011. $\mathrm{R}=0,123$

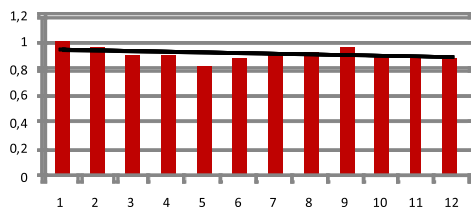

Icm CHE aval2012. $R_{-}=0,056$

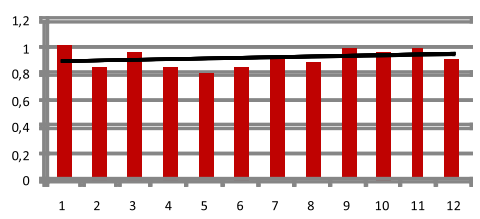

Icm $\mathrm{CHE}$ aval 2013. $\mathrm{R}=0,344$

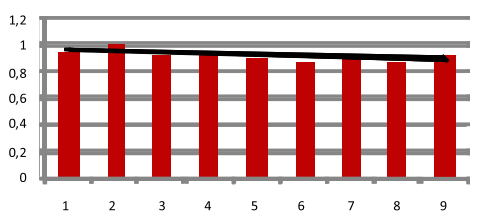

Fig. 6. Indices de condition (Icm) du chevaine lors de chaque échantillonnage à l'amont et à l'aval du CNPE au cours des six années d'étude.

Fig. 6. Annual condition index (Icm) of chub upstream and downstream of the NPP for each sampling campaign during the 6 monitoring years. 


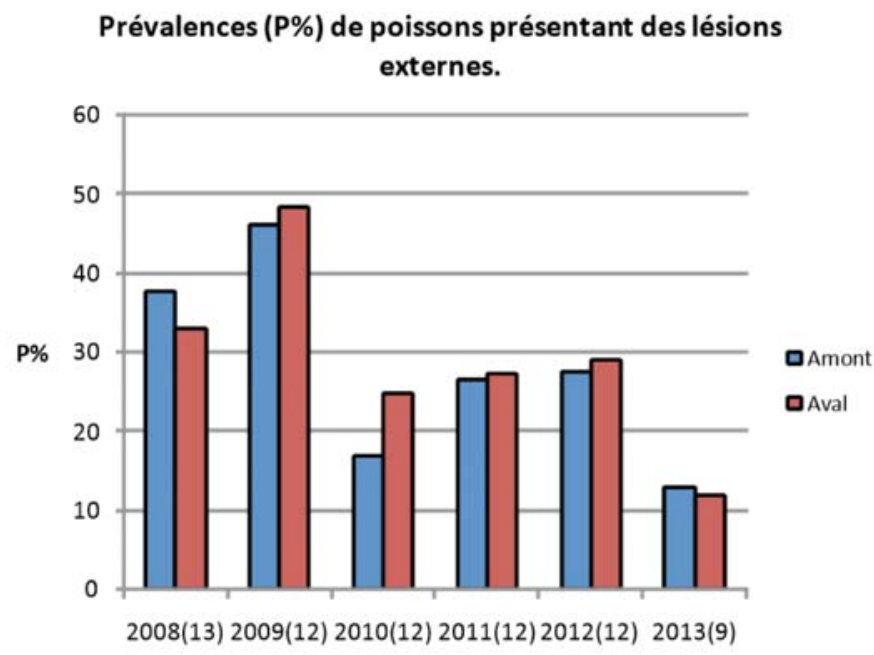

Fig. 7. Prévalences $(\mathrm{P} \%)$ annuelles de poissons présentant des lésions externes à l'amont et à l'aval du CNPE.

Fig. 7. Annual prevalence (P\%) of fish with external lesions upstream and downstream of the NPP.

lesquels les métacercaires de $B$. polymorphus.

\subsubsection{Indice pathologique}

Les différentes valeurs de cet indice (IpG) correspondent à une classe de qualité codifiée par un code couleur afin d'en faciliter l'interprétation (cf. précédemment). La qualité «précaire » (couleur jaune) est largement dominante quelle que soit la station (Fig. 10). Quelques valeurs se situent en qualité " dégradée » (couleur orange) en 2008 et 2009. Inversement, quelques valeurs sont synonymes de qualité "bonne " (couleur verte) en 2010 (amont) et 2013 (amont et aval).

Au cours de l'étude, l'IpG n'est pas significativement différent à l'amont et à l'aval du CNPE (respectivement
0,414 et 0,$426 ; \mu<\mu_{95 \%}$ soit 0,041 $<1,65)$. Aucun « effet CNPE " n'est détecté.

Lors d'une comparaison des IpG annuels entre l'amont et l'aval, aucune différence significative n'apparaît (test de Mann Whitney ; Umin $>U(0,6)$ soit $16>5$ ) (Fig. 11).

Une tendance à la baisse tout juste significative de cet indice est notée au long de la chronique d'étude $\left(R^{2}=\right.$ 0,487 ) (Fig. 12). Cependant, les Ipg sont bien significativement différents lors d'une comparaison interannuelle.

Deux outils sont utilisés pour évaluer la qualité des milieux et la santé des poissons : les Codes pathologie et l'indice pathologique.

L'outil « Codes pathologie » repose sur des descripteurs pathologiques, les lésions, et des descripteurs biologiques, les parasites externes. Cet 


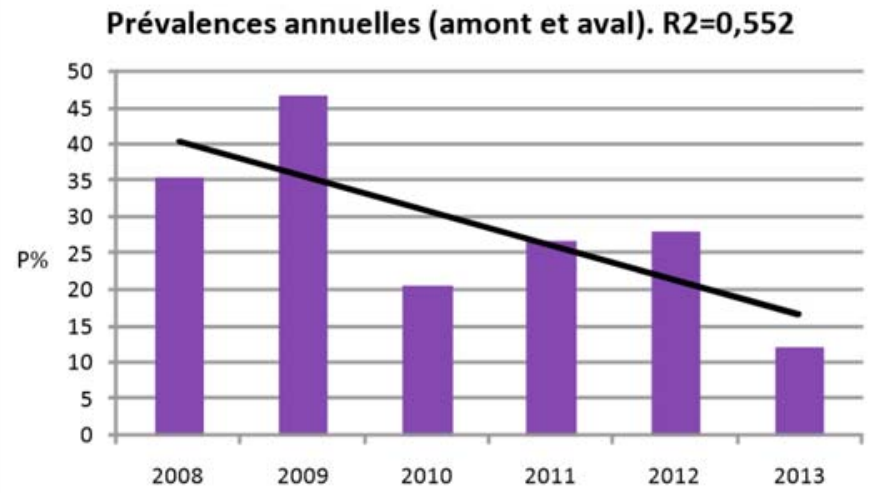

Fig. 8. Prévalences $(\mathrm{P} \%)$ annuelles (amont + aval) de poissons présentant des lésions externes.

Fig. 8. Annual prevalence (P\%) (upstream+downstream) of fish with external lesions.

outil vise à observer, décrire et quantifier la sévérité des principales anomalies et des parasites externes des poissons visibles à l'œil nu. Il permet aussi d'interpréter les données en termes de qualité de l'habitat. Toutefois, l'interprétation des données ne repose que sur un seul paramètre : la prévalence des poissons présentant des lésions ou des parasites. Et, en aucun cas, elle ne prend en compte ni le critère " sévérité / abondance ", ni l'importance de la signification écopathologique des lésions.

En revanche, l'indice pathologique prend en compte ces trois paramètres : la prévalence, mais aussi l'intensité et la signification écopathologique des lésions. De ce fait, il permet d'évaluer de façon objective la condition des populations piscicoles de différents habitats et d'établir des comparaisons intra- et interspécifiques, mais également inter-stationnelles et spatio-temporelles.

L'application systématique de l'indice pathologique permet donc de suivre et de comparer l'évolution de la condition des poissons entre, d'une part, les stations amont et aval et, d'autre part, d'une année sur l'autre (Elie \& Girard, 2014).

3.2.5 Corrélation entre prévalence des lésions et variables hydroclimatologiques

Directement et d'une manière générale, la température agit à la fois sur la sensibilité de l'hôte et sur la présence et la virulence des organismes pathogènes. L'élévation de la température de l'eau entraîne par exemple une augmentation de la fréquence de la maladie érosive des nageoires (Möller, 1979). De leur côté, les débits d'étiage et les vitesses de courants faibles favorisent les infections chez les poissons et la présence de parasites dans les systèmes aquatiques, et notamment dans les rivières. Ils sont également propices à la croissance des macrophytes. Ils créent aussi un milieu favorable à la prolifération de Gastéropodes, hôtes intermédiaires des Trématodes. 


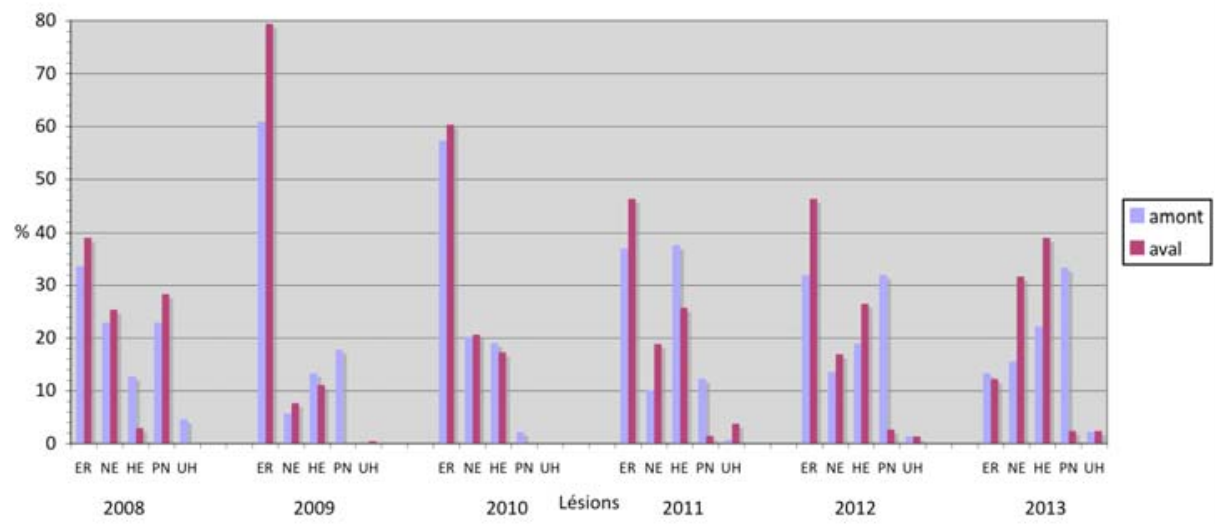

Fig. 9. Pourcentages des cinq principaux types de lésions sur les poissons présentant des lésions.

Fig. 9. Percentage of five main types of lesions on fish with lesions.

La température et I'hydrodynamisme agissent aussi de façon indirecte en tant que facteurs de stress. Chez les poissons, le stress affecte de nombreuses fonctions comme la croissance, la reproduction, l'immunité, la respiration et le métabolisme.

Or, les perturbations métaboliques, principalement la dégradation du métabolisme général et des fonctions de défense, jouent un rôle dans l'apparition des lésions tégumentaires en favorisant l'action directe des polluants et la sensibilité aux infections microbiennes.

En effet, le stress perturbe et tend à dégrader l'état physiologique des organismes en affaiblissant notamment leurs défenses immunitaires. Les poissons sont alors prédisposés aux infections secondaires provoquées par des agents potentiellement pathogènes (champignons, parasites, bactéries et virus) naturellement présents dans le milieu.
Les valeurs annuelles des prévalences n'étant pas significativement différentes entre les stations amont et aval, le test de corrélation est effectué en regroupant l'ensemble des données amont et aval. II en résulte que les prévalences ne sont pas significativement corrélées aux débits et aux températures (valeurs tendancielles pour les deux variables) ( $R^{2}$ respectivement 0,006 et 0,009 ) (Fig. 13).

\subsubsection{Corrélation entre indice pathologique et variables hydroclimatologiques}

À l'instar des prévalences, les valeurs annuelles des IpG n'étant pas significativement différentes entre les stations amont et aval, le test de corrélation est effectué en regroupant l'ensemble des données amont et aval. Et comme pour les prévalences, il en résulte que les IpG ne sont pas 
Indices pathologie globaux (IpG) des poissons de la station amont de 2008 à 2013.

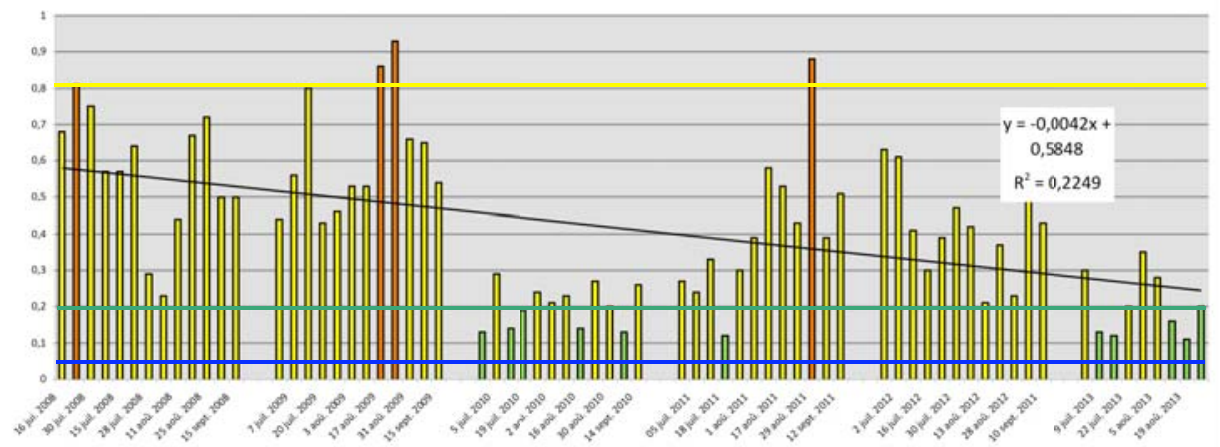

Indices pathologie globaux (IpG) des poissons de la station aval de 2008 à 2013.

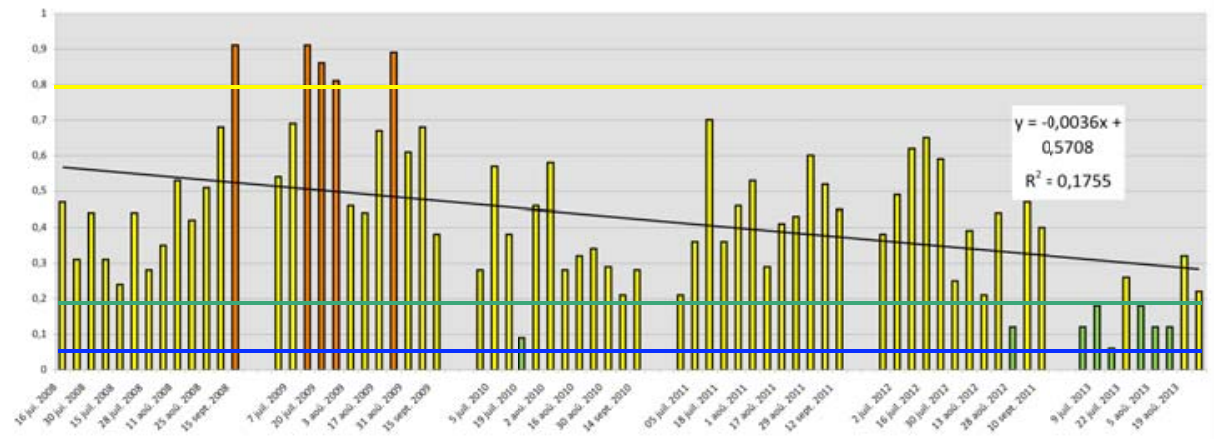

Fig. 10. Indices pathologie globaux (IpG) avec couleur des classes de qualité pour chaque échantillon et indication des limites de celles-ci.

Fig. 10. Global pathology index (IpG) with color quality classes for each sample and indication of their limits.

significativement corrélées aux débits et aux températures (valeurs tendancielles pour les deux variables) ( $R^{2}$ respectivement 0,000 et 0,005 ) (Fig. 14).

\section{DISCUSSION - CONCLUSION}

La surveillance sanitaire des populations piscicoles réalisée en amont et en aval du CNPE du Tricastin depuis 2005 a été initiée dans le cadre du "Programme de surveillance en situations exceptionnelles " des Centres Nucléaires de Production d'Électricité (CNPE) dans le cas d'un aléa climatique impactant la production d'électricité. Elle s'appuie sur des descripteurs biologiques pertinents et validés : indice de condition, prévalence des lésions et indice pathologique (Elie \& Girard, 2014). Au cours de la période d'étude (2008-2013), on observe des fluctuations plus ou moins importantes de ces indicateurs, inter- et intrastionnelles. 


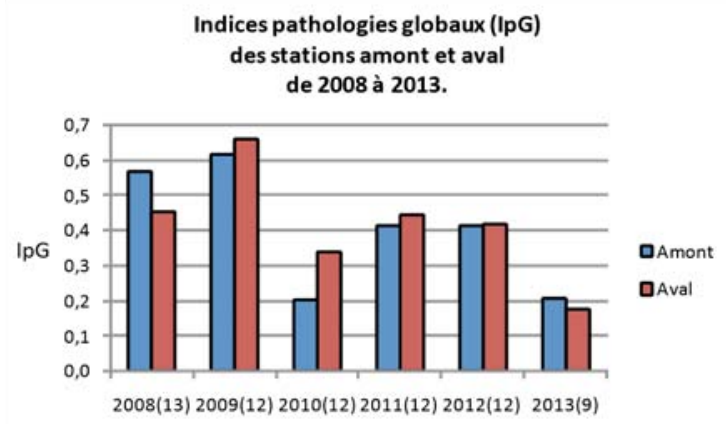

Fig. 11. Indices pathologies globaux (IpG) annuels à l'amont et à l'aval du CNPE.

Fig. 11. Annual global pathology index (IpG) upstream and downstream of the NPP.

Les valeurs de l'indice de condition varient principalement en fonction de la température de l'eau qui semble influer négativement sur ce paramètre. Effectivement, en dessous ou au-delà d'un certain seuil, les valeurs de cet indice diminuent. D'après Souchon \& Tissot (2012), les températures optimales pour le chevaine et le gardon au stade adulte sont respectivement égales à $24^{\circ} \mathrm{C}$ et $25^{\circ} \mathrm{C}$.

L'absence de différence significative des indices de condition moyens entre l'amont et l'aval, que ce soit pour le chevaine ou pour le gardon, peut s'expliquer par une différence de température pas assez marquée entre les deux stations $\left(\Delta T=1,3{ }^{\circ} \mathrm{C}\right.$ en moyenne, soit $21,3 \mathrm{C}$ à l'amont et $22,6{ }^{\circ} \mathrm{C}$ à l'aval). En revanche, les déplacements potentiels des poissons sur chaque station pendant les périodes d'échantillonnages peuvent masquer les conséquences des différences de température et donc d'indices de condition. Le remplacement d'une partie des populations amont et aval ainsi que les mortalités engendrées par l'échantillonnage luimême (individus conservés pour analyses) ne facilitent probablement pas des résultats marqués.

La température est un facteur physique essentiel qui régit le métabolisme et les fonctions physiologiques des poissons, chaque espèce piscicole disposant d'un preferendum thermique.

Les fluctuations de l'Ic, notamment sa tendance à la baisse, peuvent s'expliquer soit par la diminution de la ressource trophique due aux variations hydrologiques ou thermiques du milieu, soit - hypothèse plus probable - par une diminution du métabolisme basal des poissons lorsque les températures de l'eau s'éloignent de leur préférendum thermique. Plus on s'éloigne de cette plage thermique optimale, plus les activités métaboliques, biochimiques et physiologiques des individus sont affectées. Ces dernières ayant un coût énergétique non négligeable, les poissons se mettent alors plus ou moins au ralenti en fonction de leurs exigences thermiques. Il est également 


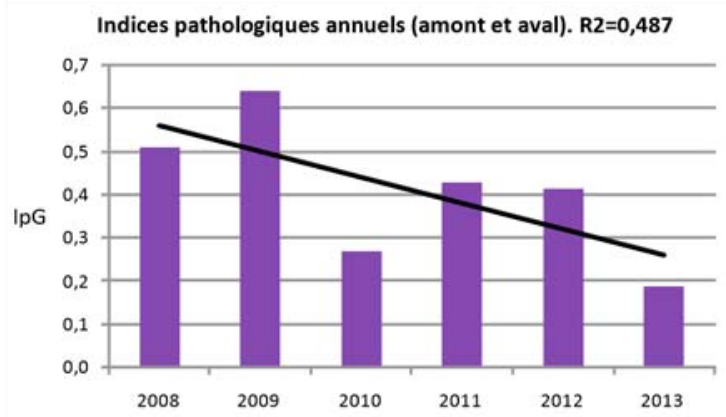

Fig. 12. Indices pathologies globaux (IpG) annuels (amont + aval).

Fig. 12. Annual global pathology index (IpG) upstream+downstream.

possible d'observer une variation de I'Icm en fonction du bilan énergétique des individus par le biais du rapport entre les apports trophiques et les dépenses physiques. La baisse quasi systématique des $\mathrm{Icm}$ des chevaines à la station amont au cours de chaque année pourrait ainsi être engendrée par la présence de l'usine-écluse de l'aménagement CNR de Montélimar. La présence de cet obstacle situé à seulement quelques centaines de mètres en amont de la zone d'échantillonnage de la station amont est une différence marquante entre les deux stations. On peut supposer que les chevaines migrent préférentiellement à l'aval de l'ouvrage au fil de la saison estivale pour trouver des températures plus adéquates à leur physiologie. La densité numérique probablement de plus en plus élevée au cours de l'été pourrait affecter les $\mathrm{Icm}$ des chevaines en augmentant la compétition alimentaire par rapport à une ressource trophique a priori constante. De plus, la vitesse du courant la plupart du temps assez élevée sur plusieurs centaines de mètres à l'aval de l'usine-écluse, alliée à un manque de zone refuge aux abords des berges, pourrait renforcer la baisse des $\mathrm{Icm}$ en augmentant les dépenses physiques des chevaines.

Les différences d'Icm des chevaines entre les stations amont et aval au fil des années semblent assez aléatoires et sans règle bien précise. La multiplicité et les variations des paramètres non contrôlés entre les deux stations ne facilitent pas l'interprétation : différences de la ressource trophique, des compétitions inter-spécifiques, des vitesses de courant, du nombre de zones de repos, de la densité des prédateurs (poissons ou oiseaux piscivores, etc.)...

Enfin, l'absence de corrélation significative des Icm des chevaines au fil des années ne permet pas de détecter de tendances particulières liées, par exemple, au réchauffement climatique.

L'absence de différence significative entre les prévalences des lésions externes moyennes des poissons entre les stations amont $(29,1 \%)$ et 

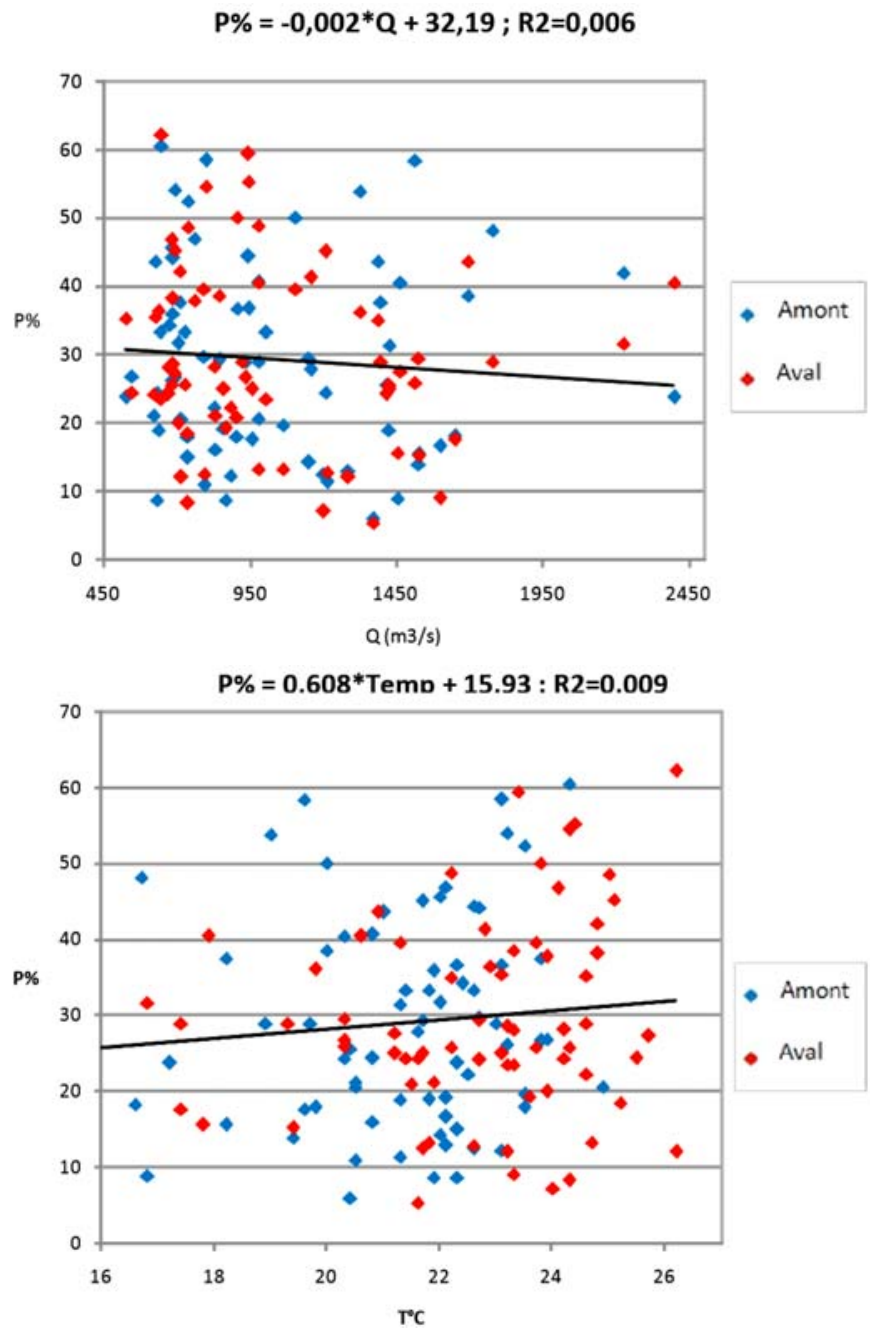

Fig. 13. Corrélation prévalences des lésions vs variables de milieu débit et température.

Fig. 13. Corrélation prevalences of lesions vs environmental variables discharge and température.

aval $(29,4 \%)$ est avérée autant globalement au cours des 6 années de l'étude que lors de chacune des années. Une différence de température moyenne de $+1,3^{\circ} \mathrm{C}$ en faveur de la station aval n'engendre pas de différence notable de prévalence de lésions. La station aval semble trop éloignée du rejet thermique (une quinzaine de kilomètres) et, par voie de conséquence, la dilution trop importante pour éventuellement espérer détecter un « effet CNPE ». Une réelle station " aval proche » aurait été plus pertinente. Toutefois, la situation de la station retenue est liée à la difficulté de 

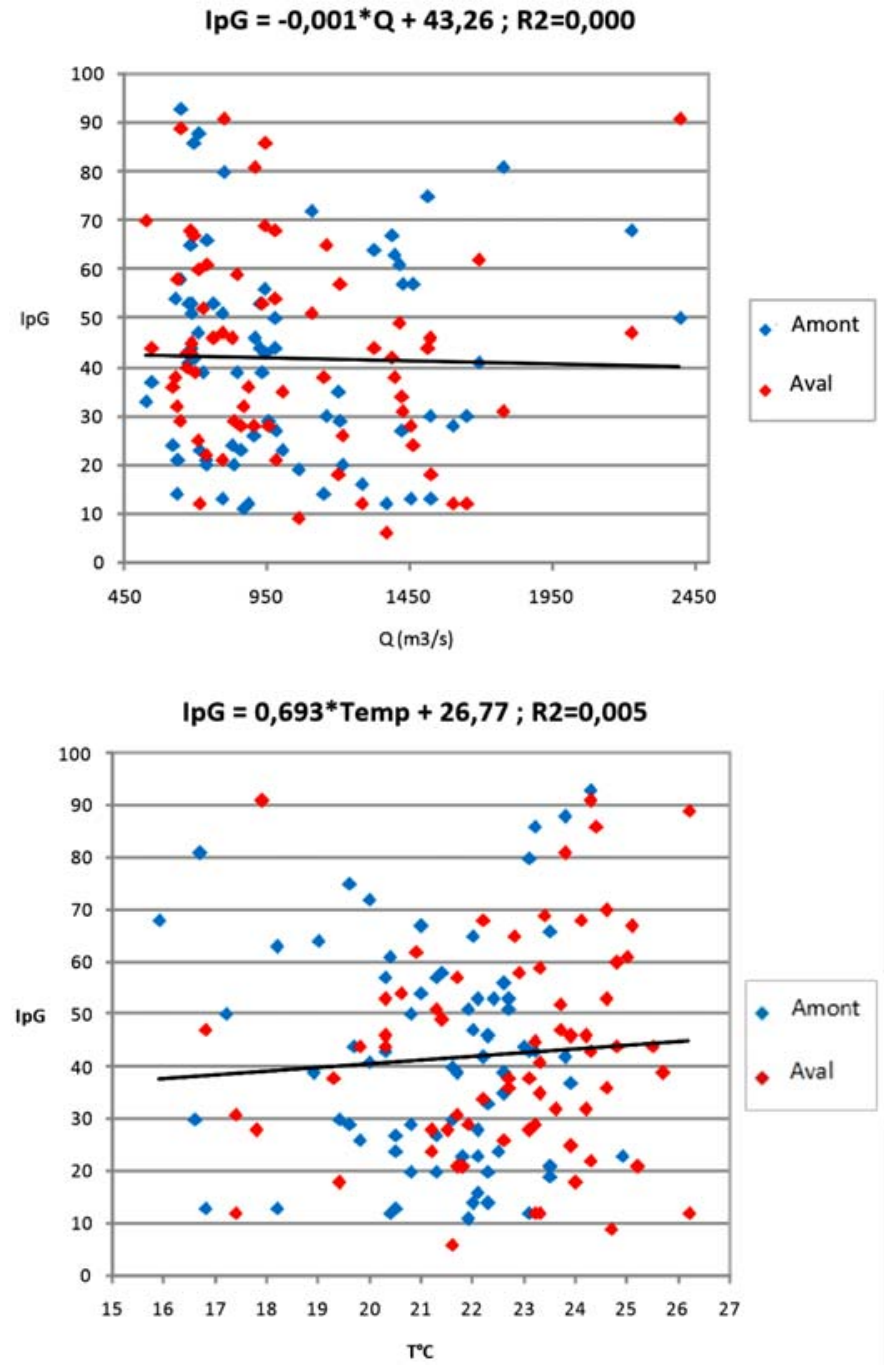

Fig. 14. Corrélation Indices pathologie globaux vs variables de milieu débit et température.

Fig. 14. Corrélation global pathology index vs environmental variables discharge and température.

trouver les espèces piscicoles recherchées et en quantités suffisantes dans un canal artificialisé et aux berges bétonnées comme l'est le canal de Donzère, mais aussi au risque d'épuiser un tel milieu au cours d'une période de 2 à 3 mois.
La cause de la baisse significative des prévalences au fil des 6 années de l'étude, que ce soit à l'amont ou à l'aval, est probablement multifactorielle et peu aisée à appréhender. Elle ne semble pas liée à une évolution de la température ou des débits au fil des 
Températures tendancielles moyennes $\left({ }^{\circ} \mathrm{C}\right) \cdot \mathrm{R} 2=0,062$

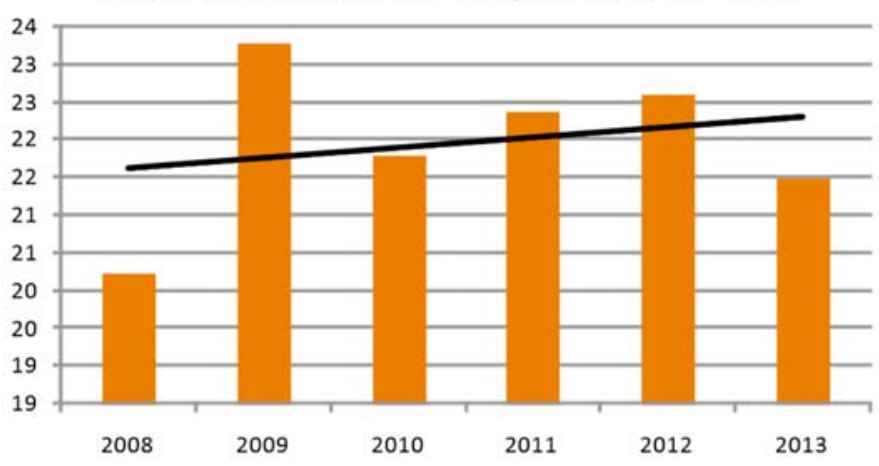

Débits tendanciels moyens (m3/s). R2=0,008

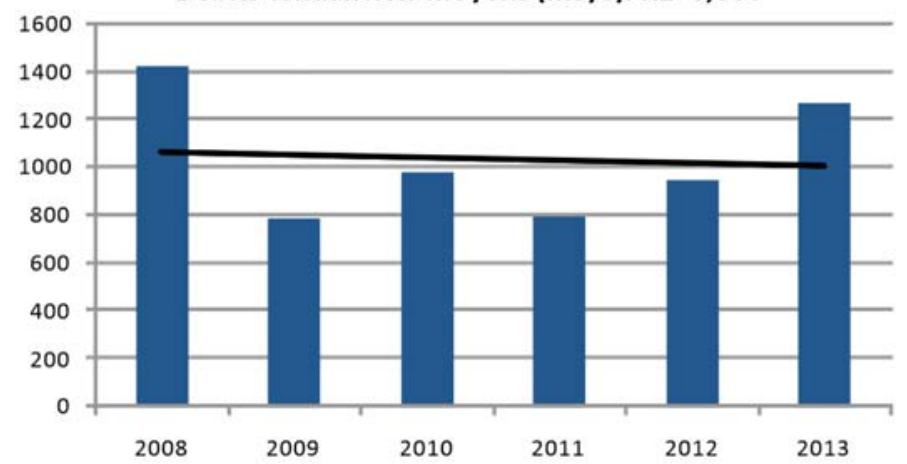

Fig. 15. Températures et débits tendanciels moyens.

Fig. 15. Average temperature and discharge 7 days before sampling.

années puisque l'augmentation (températures; $R^{2}=0,062$ ) ou la diminution (débits; $R^{2}=0,008$ ) n'est pas significative (Fig. 15).

Cinq types de lésions pathologiques et parasitaires affectent respectivement $97 \%$ (amont) et $96 \%$ (aval) des poissons : érosions, hémorragies, ulcères, nécroses et points noirs.

Les érosions, les hémorragies et les ulcères hémorragiques sont des processus pathologiques en cours d'évolution, alors que les nécroses constituent un processus en fin d'évolution ou ayant fini d'évoluer (Aubert, 1982). Ces lésions externes, cutanées ou branchiales, sont l'expression clinique de phénomènes pathologiques multiples et variés : maladies infectieuses, le plus souvent, mais aussi parasitisme, pollutions chimiques, prédation, irritations,...

Les points noirs, quant à eux, sont la conséquence de l'implantation dans le derme et le muscle des poissons de larves d'un ver Trématode, Bucephalus polymorphus. Appelées métacercaires, ces larves vont franchir la barrière cutanée, puis migrer et s'enkyster dans les tissus profonds, provoquant des lésions parfois importantes de type nécrotique et hémorragique qui 
peuvent entraîner la mort des poissons. Ce fut notamment le cas au cours de l'été 2008.

En l'absence avérée de microparasitisme externe, il est fortement probable que les lésions observées relèvent plus d'un processus purement pathologique que toxique ou chimique, provoqué très probablement par des bactéries opportunistes dont l'action pourrait être, elle aussi, thermodépendante.

Par ailleurs, même si la prévalence de poissons présentant des lésions externes - de l'ordre de $30 \%$ - paraît élevée par rapport à certaines d'entre elles observées dans d'autres études similaires réalisées en France, la très forte proportion de lésions de faible intensité tend à prouver que les processus pathologiques observés traduisent globalement plus une action ponctuelle et de faible amplitude qu'un phénomène de grande ampleur, type épidémie.

L'indice pathologique (IpG) permet d'évaluer de façon objective la condition des populations piscicoles de différents habitats et d'établir des comparaisons intra- et interspécifiques, mais également inter-stationnelles et spatio-temporelles.

Comme pour les prévalences des lésions externes, l'absence de différence significative des IpG moyens globaux et annuels entre les stations amont et aval ne permet pas de mettre en évidence un « effet CNPE » sur la santé des poissons.

Entre 2008 et 2013, les valeurs de cet indice ont tendance à décroître de façon significative sur les deux stations, traduisant ainsi une amélioration sensible et progressive de la condition sanitaire des poissons.

Cependant, aucune corrélation significative entre l'indice pathologique et le débit et la température n'apparaît sur les deux stations.

Au final, les résultats de ce suivi montrent qu'une élévation de température moyenne de $1,3^{\circ} \mathrm{C}$ n'a pas d'effet significatif sur les différents indices utilisés et, donc, sur l'état de santé des poissons. Le paramètre « débit » (variation de débit, débit d'étiage, crue) peut aussi représenter un facteur de stress potentiel pour les poissons. En effet, plusieurs études, expérimentales et de terrain, ont montré qu'il pouvait agir sur l'intégrité des individus en provoquant notamment des érosions cutanées et branchiales (par exemple par excès de matières en suspension dans l'eau suite à des crues ou des marnages importants). Cela n'a toutefois pas été vérifié au cours de ce suivi de 6 années, le pourcentage de poissons présentant des lésions externes étant bas (< $15 \%$ ) en 2013 , année où l'hydrologie est élevée et la température basse, et, inversement, élevé ( $\geq 50 \%$ ) en 2009 où l'hydrologie est basse et la température élevée.

II faut également souligner que les lésions externes observables chez les poissons sont une des réponses à des actions diverses et variées (manifestation clinique de maladie, chocs, traumatismes ou irritations, stress, prédation, parasitisme, exposition à des micropolluants,...). Or, les lésions externes constituent généralement l'une des étapes ultimes du poisson lorsque celui-ci est confronté à des processus spécifiquement pathologiques, 
tels que les organismes pathogènes ou les micropolluants (Elie \& Girard, 2014). Ainsi, les lésions observées dans le cadre de cette étude sont plus le fait d'atteintes pathologiques mineures, essentiellement provoquées par des bactéries opportunistes, que toxicologiques.

Enfin, avec l'effet de plus en plus prégnant du changement climatique global sur les peuplements aquatiques des hydrosystèmes continentaux (Daufresne et al., 2003 ; Daufresne \& Boët, 2007 ; Durance \& Ormerod, 2009 ; Fruget et al. 2015), une réflexion sur la prise en compte et le suivi des effets potentiels du réchauffement engendré sur le développement des maladies des poissons et sur le comportement des espèces résidentes et migratrices semble nécessaire. D'autant plus que non seulement nous disposons d'indicateurs pathobiologiques pertinents, fiables, peu onéreux et facilement utilisables sur le terrain (Indice de condition, Codes pathologie, Indice pathologique), mais aussi que d'autres descripteurs (hématologiques et histologiques) sont également susceptibles d'être intégrés dans ces outils de diagnostic et de surveillance de l'état de santé des populations piscicoles et de la qualité des milieux qui les hébergent.

\section{REMERCIEMENTS}

Cette étude est financée par Electricité de France (EDF) dans le cadre du suivi environnemental du CNPE du Tricastin. Merci aux différents collègues d'ARALEP qui collaborent chaque semaine aux pêches de collecte des poissons examinés. Les données hydrologiques et thermiques proviennent des enregistrements EDF Tricastin.

\section{RÉFÉRENCES BIBLIOGRA- PHIQUES}

Aubert M. et al., 1982. Recherches sur les nécroses des poissons. CERBOM, rapport final de synthèse 1977-1982. $35 \mathrm{p}$.

Baumann P.C., Smith S.B. \& Metcalfe C.D., 1996. Linkages between chemical contaminants and tumors in benthic Great Lakes fish. J. Great Lakes Res. 22 (2) : 131-152.

Bogé G., Roche H. \& Houvet D., 1991. Les indicateurs physiologiques de toxicité en milieu marin. Océanis, 17 (4) : 351365.

Carrel, G., Archambaud, G., Colombet, X., Testi, B. \& Favriou, P., 2013. Suivi hydrobiologique du Rhône. CNPE du Tricastin. Résultats de l'année 2013. Rapport à EDF CNPE Tricastin. U.R. Hydrobiologie, Irstea, Aix-en-Provence, $76 \mathrm{p}$.

Daufresne M. \& Boët P., 2007. Climate change impacts on structure and diversity of fish communities in rivers. Global Change Biology 13 : 2467-2478.

Daufresne M., Roger M.C., Capra H. \& Lamouroux N., 2003. Long-term changes within the invertebrate and fish communities of the Upper Rhône River: effects of climatic factors. Global Change Biology 10 : 124-140.

Durance I. \& Ormerod S.J., 2009. Trends in water quality and discharge confound long-term warming effects on river macroinvertebrates. Freshwater Biology 54 : 388-405.

Elie P. \& Girard P., 2014. La santé des poissons sauvages : les Codes pathologie, 
un outil d'évaluation. Association Santé Poissons Sauvages Ed., 286 p.

Fruget J.F., Jézéquel C., Archambaud G., Dessaix J. \& Roger M.C., 2015. Longterm effects of global and local changes on benthic macroinvertebrate communities in multi-stressed large rivers: the example of the Rhône River during the last 30 years. Knowl. Manag. Aquat. Ecosyst. 416, 29.

Girard P., 1998. Le poisson sentinelle des milieux aquatiques : pertinence et optimisation des indicateurs sanitaires. Bull. Fr. Pêche Pisci. 300-351 : 429-443

Girard P. \& Elie P., 2007a. Manuel d'identification des principales lésions anatomomorphologiques et des principaux parasites externes des anguilles. CEMAGREF / Association Santé Poissons Sauvages Eds, $67 \mathrm{p}$.

Girard P. \& Elie P., 2007b. Les mécanismes d'induction de stress chez les poissons des eaux douces, estuariennes et marines et leurs répercussions : proposition d'un indice anatomo- morphologique et parasitaire externe pour l'évaluation de l'état de santé des poissons. Pro- gramme Seine Aval. Étude CEMAGREF $\mathrm{n}^{\circ} 113,130 \mathrm{p}$.

Girard, 2009. Influence de la température sur l'état sanitaire des poissons. Rapport de synthèse. Convention EDF R\&D, $73 \mathrm{p}$.

Möller H., 1979. Geographical distribution of fish diseases in the NE Atlantic. Meeresforschung 27: 217-235

Nounou P. et al., 1980. Ulcérations des poissons et mammifères marins pêchés dans les eaux côtières françaises. CNEXO, Rapports scientifiques et techniques $n^{\circ} 43,94 \mathrm{p}$.

Nounou P., 1985. Etat sanitaire des poissons d'intérêt commercial pêchés dans les eaux côtières françaises. IFREMER, Rapport scientifique et technique $\mathrm{n}^{\circ} 2$, $297 \mathrm{p}$.

Souchon Y. \& Tissot L., 2012. Synthesis of thermal tolerances of the common freshwater fish species in large Western Europe rivers. Knowl. Manag. Aquat. Ecosyst. 405, 03.

Travade F., 1975. Influence du réchauffement des eaux continentales sur les maladies des poissons. Etude bibliographique. EDF. Rapport F41-75 $\mathrm{n}^{\circ} 22$, ARD F4D02, $59 \mathrm{p}$. 